\title{
A tensão naturalismo/normativismo no campo da definição da doença*1
}

Paula Gaudenzi*2

Vemos uma tendência da psiquiatria contemporânea de reduzir a experiência subjetiva da doença a achados neurofisiológicos. Na contramão desse movimento estão os trabalhos de Bill Fulford e Georges Canguilhem. O primeiro aposta na precedência da illness sobre a disease; Canguilhem defende a ideia de que o pathos precede o logos e ambos afirmam a inseparabilidade entre fatos e valores. Trazem, assim, a ênfase na dimensão sócio-simbólica da experiência subjetiva, marcando a centralidade da clínica para a interrogação sobre o sofrimento humano.

Palavras-chave: Filosofia da medicina, psiquiatria, Fulford, Canguilhem

*1 Baseado na tese acadêmica: "Normal e patológico: leituras contemporâneas", 2014, IMS/Universidade do Estado do Rio de Janeiro - UERJ (Rio de Janeiro, RJ, Br).

${ }^{*}$ Fundação Oswaldo Cruz - Fiocruz (Rio de Janeiro, RJ, Br). 


\section{Introdução}

A pergunta se a ciência pode nos esclarecer $o$ que é a doença ou se ela é necessariamente relativa a valores compartilhados culturalmente pertence ao debate tanto em relação às doenças físicas como às doenças mentais, mas é trabalhada especialmente no campo de estudo das doenças mentais. Para o historiador Charles Rosenberg (2006), a negociação do estatuto nosológico de diversas doenças psiquiátricas tem sido cada vez mais frequente nas últimas décadas, o que coloca em questão a legitimidade social e epistemológica desses diagnósticos. Tal questão atormenta filósofos, profissionais de saúde e pacientes, pois o dilema da natureza da doença psiquiátrica e da (i)legitimidade quanto ao estatuto ontológico da mesma constitui o princípio-chave que organiza as decisões clínicas a serem tomadas.

$\mathrm{Na}$ psiquiatria, o problema da fronteira entre o normal e o patológico se relaciona fundamentalmente com o problema da presença de valores na definição da doença. Na tentativa de responder se o transtorno mental é algo biologicamente real ou se é necessariamente uma classificação baseada em valores morais, filósofos da psiquiatria apelam para a teoria evolucionista - a qual tem um papel proeminente nas abordagens naturalistas e híbridas sobre a doença mental. Apesar do entendimento de que o transtorno mental é um desvio de uma norma e portanto um valor - os naturalistas defendem que a norma relevante é uma norma biológica, que pode ser descoberta por meio do método científico. Desse prisma, os casos paradigmáticos dos transtornos mentais, como a esquizofrenia e a depressão, são compreendidos como resultado de um mecanismo psicológico que falha em se conformar com a norma natural (De Block \& Adriaens, 2011). 
O principal debate filosófico sobre a doença mental não é exatamente sobre a sua existência, mas, sobretudo, sobre como oferecer uma definição científica ou objetiva e qual seria a contribuição dos fatores subjetivos e valorativos em sua definição. Georges Canguilhem (1995) foi um dos autores que realizou uma análise rigorosa dos conceitos de saúde e doença na tentativa de superar a dicotomia entre fatos e valores, contribuindo significativamente para a análise do estatuto epistemológico desses conceitos médicos. Analisaremos o trabalho do autor, marcando a atualidade de seu pensamento em diálogo com o trabalho de Bill Fulford, psiquiatra britânico e professor de Filosofia e Saúde Mental na faculdade de filosofia da Universidade de Warwick, no Reino Unido. A interlocução parece especialmente relevante se considerarmos que é no campo da psiquiatria que se encontram as maiores controvérsias sobre a fronteira entre o normal e o patológico e sobre o papel dos valores nesta discussão.

\section{A atualidade do debate entre o normal e o patológico}

Vemos hoje um predomínio da literatura anglo-saxônica na discussão filosófica sobre os conceitos médicos. Apesar da maioria dos autores anglofônicos não dialogarem com Canguilhem, identificamos que eles compartilham com o filósofo francês a convicção de que a análise filosófica pode contribuir para o esclarecimento dos conceitos médicos.

Trinta anos após a publicação do trabalho seminal de Canguilhem, Ensaios sobre alguns problemas concernentes ao Normal e ao Patológico, uma literatura filosófica, principalmente anglo-saxônica, tomou corpo e assumiu o desafio de trabalhar a problemática concernente à definição dos conceitos de saúde e doença. Teve início uma controvérsia, descrita na maioria das vezes como um debate entre "naturalistas" e "normativistas".

A abordagem naturalista em medicina - também chamada de empirista, objetivista ou positivista - sustenta a existência de um fundamento racional, valorativamente neutro para se falar em saúde e doença. Diferenciam-se, assim, dos normativistas que afirmam que uma explicação satisfatória da saúde é intrinsecamente carregada de valores e, portanto, contextual. A meta dos naturalistas é mostrar que a distinção entre o normal e o patológico não é inventada, mas factual, seguindo a tradição objetivista da explicação da doença e da normalidade.

No livro The Construction of Social Reality, John Searle (1995) faz uma distinção entre as noções de objetividade e subjetividade útil para elucidar o debate. Searle propõe dois sentidos da distinção entre objetivo e subjetivo: o epistêmico e o ontológico. No epistêmico, objetivo e subjetivo são predicados de julgamentos. Isto 
é, o que distingue os dois tipos de julgamentos é se a verdade ou falsidade de cada um depende ou independe das crenças individuais, das atitudes e dos sentimentos do observador. No sentido ontológico, por sua vez, objetivo e subjetivo são tipos de entidades que correspondem a diversos modos de existência. O que os distingue é a existência dependente ou independente de nossa representação. Neste sentido, um pedaço de ouro é uma entidade ontologicamente objetiva enquanto a dor é uma entidade ontologicamente subjetiva.

Os sentidos epistêmico e ontológico, para Searle, fundamentam o pensamento naturalista em matéria de saúde e doença e estabelecem as bases para a diferença entre naturalismo e normativismo (Guerrero, 2011). Compreender o estado de saúde como função de um fato ontologicamente subjetivo é fazer da doença algo que só existe na presença de seres humanos. Nessa abordagem, a saúde é um evento dependente da experiência subjetiva, de normas e valores morais. O naturalismo, ao contrário, aborda a saúde e a doença sem fazer referência a fatos ontológicos e julgamentos epistêmicos subjetivos. O julgamento sobre a doença, portanto, seria ou deveria ser objetivamente falso ou verdadeiro, no sentido de Searle. ${ }^{1}$

Em relação aos normativistas, a démarche é outra. A corrente normativista, também chamada de subjetivista ou construtivista, afirma que tudo que existe só faz sentido dentro de um universo linguístico com regras de consistência de entendimento e dentro de um universo genealógico - de uma história de saberes, de poderes, de determinações éticas ou estéticas. Assim, os normativistas diferem substancialmente dos naturalistas, ao considerar que o contexto epistêmico é indissociável do contexto histórico-cultural. Desta forma, podemos dizer que o normativismo é uma variante do historicismo, do construtivismo e do pragmatismo que defende que o sentido que damos às coisas é dependente do background cultural, genealógico, gramático, epistemológico e histórico que possuímos.

Identificamos, porém, que a maioria dos autores não se enquadra completamente na polarização proposta. A distinção normativismo/naturalismo parece mais razoável como artefato heurístico destinado a identificar os principais fundamentos

${ }^{1}$ A crença na existência de um mundo real independente das representações, paradigmas ou esquemas conceituais é criticada por exemplo por Richard Rorty. Rorty ataca a "teoria da verdade como correspondência" e nega a independência representacional, afirmando que não há como falar da realidade para além das relações descritivas que temos com ela. Nega, portanto, o conceito de realidade objetiva e duvida da utilidade de qualquer teoria da verdade nessa acepção. Para ele, verdadeiro é apenas um termo de recomendação que usamos para elogiarmos as crenças que pensamos como boas de acreditar, tratando a verdade em termos semânticos. Para um aprofundamento do tema, ver: Ghiraldelli (1999). 


\section{OBSERVANDO A PSIQUIATRIA}

utilizados pelos defensores dessas abordagens e a analisar o papel das normas e valores na conceitualização da doença. Parece-nos que o debate não se limita a afirmar ou negar a presença de valores na definição dos conceitos médicos, mas também se estrutura de forma a problematizar a relação fato/valor e a relação lógica entre os componentes biológicos e práticos na concepção de doença.

\section{Bill Fulford: aproximações canguilhemianas}

Partindo do método da filosofia analítica, que visa descrever a "geografia lógica" dos conceitos em determinada área do discurso (Nordenfelt, 2001), Fulford (2001) informa que a visão biológica dos conceitos de disease, em termos de distúrbios do funcionamento do corpo e da mente e a visão social sobre as concepções positivas da saúde relacionada com a competência social, são estruturalmente interdependentes. Ele propõe os valores, a illness e a falha da ação como elementos lógicos numa teoria que visa ser uma teoria ponte situada entre as teorias biológicas e sociais. A illness situa-se entre partes do corpo e estruturas sociais e, similarmente, as ações dos indivíduos estão entre o funcionamento de suas partes corporais - das quais as ações são contingentemente dependentes - e os papéis sociais, por meio dos quais, em parte, a competência das ações dos agentes é mensurada.

\section{A distinção fato/valor}

De forma geral, os naturalistas defendem uma separação radical entre fato e valor. A doença definida objetivamente é, portanto, um fato. Embora não desconsiderem a presença de valores, os naturalistas informam que os mesmos são incorporados apenas posteriormente, na prática médica (Ruse, 1997). Mas a dicotomia fato/valor não está isenta de crítica.

Ambos os autores - Canguilhem e Fulford - pretendem afirmar a interdependência ontológica entre a dimensão do fato e a dimensão do valor, contrapondose ao modelo médico tradicional. Mas vemos que enquanto Canguilhem pretende fazer do valor um fato, Fulford se empenha em afirmar a inexistência de "fatos livres de valor".

Canguilhem (1995) pretende superar a dicotomia fazendo uso da noção de normatividade vital - a capacidade que a vida possui de determinar na consciência os valores fundamentais à sobrevivência do organismo - um conceito polêmico sobretudo por supostamente incidir em uma falácia naturalista, termo cunhado pelo filósofo inglês G. E. Moore para designar o erro em associar um conceito ético com 
um conceito "natural" ou em deduzir "o que deve ser" daquilo que "é" (Blackburn, 1994). Fulford (2004; 2006), por sua vez, discorda da defesa do modelo médico tradicional de que haveria uma distinção ontológica entre a dimensão dos fatos e a dimensão dos valores e afirma que a separação é meramente gramatical.

Canguilhem sente necessidade de oferecer uma formulação mais objetiva do que a interpretação axiológica proposta pela filosofia da medicina tradicional e a contraria propondo a união entre "objetivo" e "subjetivo", a partir da imanência da normatividade vital. Apoiado em Goldstein, para quem saúde e doença sempre são normas individuais, Canguilhem reinterpreta essa ideia médica dentro do paradigma darwinista da vida. A tese é a da íntima relação entre individualidade e valor vital. Trata, portanto, da defesa de que a axiologia está enraizada nos fundamentos conceituais das ciências biológicas (Gayon, 1998).

Segundo Gayon (1998), Canguilhem quer generalizar o conceito axiológico da doença para retirá-lo do universo subjetivo da experiência pessoal. O autor, então, situa a experiência da doença no amplo contexto da vida e visa compreender melhor a relação entre medicina e biologia. Neste ponto, Fulford distancia-se de Canguilhem, pois não se debruça sobre a biologia e considera ciências médicas e ciências biológicas como empreitadas distintas.

Fulford (2004) propõe uma Medicina Baseada em Valores, uma psiquiatria filosófica e antropologicamente orientada, baseada nos valores e na narrativa. Posteriormente, Fulford preferirá o termo Prática Baseada em Valores como forma de aplicar a ideia às demais profissões da saúde. $\mathrm{O}$ autor visa oferecer uma alternativa à prática em saúde mental pautada exclusivamente nas evidências científicas. A proposta é superar a concepção ingênua da Medicina Baseada em Evidências fundamentada sobre uma visão representacionista da linguagem na qual há uma correspondência exata entre linguagem e mundo e que visa sustentar os diagnósticos médicos como "descobertas científicas" e, portanto, verdades inquestionáveis e universais. Diferentemente, para David Sackett (2001 apud Woodbridge-Dodd \& Fulford, 2004), a Medicina Baseada em Evidências comporta, além das evidências das pesquisas e da prática clínica, os valores dos pacientes.

Woodbridge-Dodd \& Fulford (2004) não negam as evidências e referem que a Prática Baseada em Valores se desenvolveu para estar ao lado da Prática Baseada em Evidências. Os autores propõem o modelo "fato mais valor" que, à maneira de Putnam (1999), se fundamenta em uma visão não representacionista da linguagem e assume a inseparabilidade entre as evidências e os valores. Para Fulford (2004), não há uma correspondência exata entre linguagem e realidade, pois a linguagem é indissociável dos contextos socioculturais específicos e, assim, não é possível fazer uma descrição extracultural. A dimensão dos fatos, diz, é atravessada pela dimensão dos valores e a separação entre fatos e valores existe apenas conceitualmente e não ontologicamente como defende o modelo médico tradicional. 


\section{OBSERVANDO A PSIQUIATRIA}

\section{Disease e Illness}

Fulford oferece uma alternativa à interpretação médica convencional sobre a relação entre os conceitos de disease e illness. Nesta visão, a disease é usada com conotação factual, definida o tanto quanto possível em termos de disfunção, um conceito biológico-científico supostamente livre de valor, do qual a illness é derivada. A alternativa é a teoria reversa da doença, em que a illness é um conceito logicamente anterior à disease. Fulford quer enfatizar que sua teoria valorativa reverte a relação lógica - e não causal - entre illness e disease como assumida pelo modelo médico tradicional.

Para Boorse $(1975 ; 1977)$, um dos principais representantes do naturalismo, haveria dois sentidos de "saúde", um teórico, que seria o oposto de disease, e um prático indissociável de um componente moral, que seria o oposto de illness. O funcionamento biológico e a disfunção fundamentariam a noção necessária para a teoria médica, que concerne ao patologista, enquanto a enfermidade seria relevante para a prática clínica. Nesta concepção, apenas o conceito prático é axiológico e o equívoco em considerar a saúde como um conceito essencialmente valorativo é explicado pela confusão entre os sentidos teórico e prático da doença.

Ademais, Boorse afirma a disease como o conceito primário, o conceito cientificamente estabelecido, e illness o conceito subjetivo derivado. Esta é uma diferença marcante entre naturalistas e normativistas. Os naturalistas concebem a prioridade lógica do fisiológico sobre o patológico e da fisiopatologia sobre a clínica. A defesa da prioridade lógica da fisiologia sobre a clínica ou do conceito teórico sobre o conceito prático de doença é vista em Claude Bernard (Giroux, 2011), médico francês do século XVIII, criador da definição fisiológica e experimental da doença. Para Bernard, as normas fisiológicas podem ser determinadas experimentalmente e o patológico pode ser inferido delas quantitativamente.

É precisamente a dedução do patológico, do fisiológico e a subordinação da clínica à fisiopatologia, o objeto de críticas de Canguilhem e de Fulford. Segundo Canguilhem (1995), preocupados em fazer da medicina uma "ciência propriamente dita", os naturalistas caem em um excessivo reducionismo, pois recusam a necessária negatividade da doença para o indivíduo, isto é, a experiência vivida de sofrimento e impotência, que é o objeto por excelência da "pathos-logia". Canguilhem e Fulford, ao contrário, defendem que os conceitos de saúde e doença devem ser entendidos a partir do referencial clínico e rechaçam a ideia de que o trabalho filosófico sobre estes conceitos deve ter como fundamento a medicina fisiológica.

Grande parte dos naturalistas contemporâneos reatualiza o "princípio de Broussais", informando que os fenômenos normais e patológicos, ou que a fisiologia e a patologia são idênticas, diferindo apenas quantitativamente, em termos de 
intensidade. Mas para Canguilhem e Fulford, a relação entre fisiologia/patologia e ordem/transtorno não é simples. Como mostra Canguilhem (1995), a patologia estabelece relações complexas com disciplinas afins como a fisiologia e a terapêutica. Na medicina, diz ele, existe uma confusão oriunda do termo normal designar ao mesmo tempo o estado habitual dos órgãos (normal como fato) e seu estado ideal (normal como valor).

O grande objetivo de Canguilhem é abolir a ideia de que possa haver uma medicina e uma patologia científicas, isto é, de que haveria um critério objetivo, quantitativo, de demarcação entre o normal e o patológico. A frequência estatística, segundo Canguilhem, não nos oferece subsídios para a consideração de uma norma de vida. Para o autor, "um traço humano não seria normal por ser frequente; mas seria frequente por ser normal, isto é, normativo num determinado gênero da vida..." (p. 126). Assim, o uso primeiro do termo normal é valorativo, sendo incompatível com a posição quantitativa que afirma que a patologia seria um afastamento de uma média. Este juízo de valor não pode ser universalizado, transformado em uma norma a ser seguida, pois isso ocasionaria um regime normatizante que consideraria anormais os que se desviam do padrão.

Segundo Canguilhem (1995), se a fisiologia é capaz de identificar uma doença, é à clínica que se deve esse reconhecimento. A descoberta do fato patológico não é um dado tributário primeiramente da ciência; é o produto da denúncia humana de seu mal-estar. Citando René Leriche, "saúde é a vida no silêncio dos órgãos", Canguilhem reitera que a saúde é a inconsciência do corpo, sendo sua consciência despertada pelos limites impostos pela doença. A experiência da doença rompe a imanência silenciosa entre o sujeito e o seu corpo e transforma o corpo em um "problema" que cria exigências de saber e configura necessidades de cuidado e intervenção. Assim, Canguilhem reposicionou a hierarquia entre ciência e terapêutica, afirmando que não se trata de conhecer para valorar e intervir, mas sim valorar para conhecer e intervir.

Fulford concorda com Canguilhem e problematiza a relação entre disease e illness. Para ele, é a illness que define a disease. De acordo com Fulford (1989, 1993b), a disease é a illness reconhecida, identificada em termos de sintomas e posteriormente classificada. O grupo de sintomas adquire um nome e é incluído nas classificações médicas, tornando-se um tipo de patologia. Uma vez definida a classe, outras classes de condições serão adicionadas por meio do processo de generalização.

Assim, uma vez estabelecido o que conta como illness e secundariamente elaborado o conceito de disease, é possível fazer inferências da disease à illness. Quando as causas das illnesses são estabelecidas, há predomínio de categorias de disease, as quais podem ser definidas mesmo na ausência de sintomas. Uma vez que as categorias causais de doença se tornam cada vez mais utilizadas como 
resultado do desenvolvimento da ciência médica e biológica, elas se tornam mais proeminentes no discurso médico e aparentam ser logicamente primárias. Mas a cadeia se inicia na experiência subjetiva da illness. O fluxo de sentido é da illness para a disease e não o contrário, concordando com Canguilhem.

De acordo com Fulford (1993a, 1993b), o debate sobre o que é a doença mental costuma se sustentar no pressuposto de que o conceito de "doença física" tem um significado relativamente transparente e não problemático por ser definido em termos objetivos - relativos ao funcionamento biológico - e destituídos de julgamentos de valor. Desse aspecto, a validade da noção de doença mental dependeria de ser ou não moldada para se adequar ao paradigma da doença física.

Mas o autor sugere que, assim como a doença mental, a doença física não se define exclusivamente por critérios factuais, mas também por julgamentos de valor. Não é o significado da doença mental que é problemático, mas o da doença física. As doenças mentais e as doenças físicas seriam, desse ângulo, conceitualmente similares. Ao invés de reduzir a illness mental ao físico, o autor propõe a generalização, isto é, entende que as illnesses mentais e as illnesses físicas são igualmente subespécies válidas do conceito geral illness.

A grande "sacada" de Fulford (1993b) advém da ideia de Hare de que a variação nas conotações valorativas dos termos reflete a extensão em que os critérios descritivos dos julgamentos de valor são assumidos de maneira tácita. Segundo Fulford (1993b; 2001), podemos aplicar esta ideia aos conceitos médicos. Para o autor, os termos disease e disfunção, frequentemente utilizados na teoria naturalista da doença, apesar de aparentemente descritivos, possuem elementos valorativos evidentes. A impressão de que não há valores implicados na definição da doença física é oriunda do fato de que os valores relativos ao funcionamento do organismo são amplamente compartilhados e coincidentes com a norma biológica. Uma vez que os valores implicados da doença física são mais consensuais, adquirem conotação descritiva. Diferentemente, os valores que concernem à experiência e ao comportamento humano, implicados na definição da doença mental, são muito varáveis. Estamos falando em crença, desejo, volição, emoção e outros. É razoável que as pessoas divirjam mais sobre o que é o bom funcionamento afetivo do que sobre o bom funcionamento cardíaco.

Diferentemente de grande parte dos naturalistas, Fulford considera impensável oferecer uma descrição aparentemente livre de valor da doença mental. Para que a doença mental tenha conotação igualmente descritiva depende da supressão das legítimas diferenças de valores entre as pessoas e não do estabelecimento de sólidos substratos neuropatofisiológicos. Em sua concepção, não há como analisar o que é uma vida psíquica saudável sem ter uma ideia sobre o que é uma "boa vida" ou uma "vida realizada" e essas noções são altamente subjetivas (Banzato \& Dantas, 2012). 


\section{A importância do debate para o campo da psiquiatria}

A defesa da precedência da illness sobre a disease e a fórmula lapidar de que o pathos precede o logos trazidas por Fulford e Canguilhem, respectivamente, têm importantes implicações práticas em dois pontos fundamentais. De um lado, elas permitem complexificar o debate sobre a existência das doenças mentais como fato ou como construção social. Por outro lado, elas ressaltam a centralidade da clínica atualmente renegada a um segundo plano diante das inovações tecnológicas e do crescimento vertiginoso da psiquiatria biológica.

Referente à primeira problemática, o debate sobre a existência da doença mental em geral é polarizado. Para alguns se trata de entidades com limites bem definidos biologicamente que poderiam ser descobertas e, para outros, elas seriam construções humanas arbitrárias. Aqueles que afirmam a doença mental como fato biológico reduzem a diferença entre doença física e doença mental e a entendem como falha do órgão em executar sua função biológica normal, o que é uma tendência da psiquiatria contemporânea que privilegia a dimensão biológica do ser humano, mais especificamente o cérebro, em detrimento à dimensão subjetiva (Kendler, 2008).

Para os que acreditam que doença física e doença mental devem ser explicadas por meio de diferentes referenciais assume-se que a expectativa quanto à conformidade ao padrão da espécie é contrariada no caso das doenças mentais. Entende-se que o que predomina neste caso não é um mau funcionamento de um órgão, mas um desvio de uma norma humana de funcionamento social adequado ou desejável.

Mas Fulford vai na contramão dessa tendência. Afirmando a anterioridade lógica da illness em relação à disease reduz a diferença conceitual entre doença física e doença mental. O autor propõe uma generalização, entendendo que as illnesses físicas e as illnesses mentais são subespécies do conceito geral illness e, assim, afirma a necessária consideração dos valores antropomórficos para a caracterização da doença. Dessa forma, Fulford se afasta da visão reducionista sobre o transtorno mental, pautada no determinismo biológico e nos achados fisiopatológicos que reduz o mental ao cerebral. É importante lembrar, porém, que a negação de Fulford da visão das doenças mentais como tipos naturais não corresponde à recusa às suas bases biológicas.

Enquanto para a teoria biológica a psiquiatria é vulnerável ao abuso diagnóstico porque os conceitos diagnósticos são cientificamente primitivos, no ponto de vista de Fulford a vulnerabilidade da psiquiatria ao abuso diagnóstico é oriunda da natureza relativamente aberta dos julgamentos de valor envolvidos no diagnóstico psiquiátrico. Ela é irredutível, pois reflete a diversidade dos seres humanos. Mas tal irredutibilidade é valorizada nas sociedades democráticas 
pluralistas contemporâneas, pois a variedade de considerações valorativas é vista como uma importante proteção contra a hegemonia de qualquer ponto de vista, e sua consideração é um componente essencial da boa prática clínica.

Em relação ao segundo ponto, é notável a importância da afirmação da precedência do pathos sobre o logos para o resgate da clínica na psiquiatria. Priorizar logicamente a experiência singular vivida em detrimento do saber científico universal ilustrado no (mau) uso dos manuais diagnósticos é uma forma de resistir à redução do sujeito e seu sintoma às nomenclaturas dos transtornos mentais, que os engessa em classificações identitárias universais. A existência de um disfuncionamento em relação a um ideal de saúde e bem-estar predeterminado faz com que a prática clínica dirija-se a um conjunto de normas e à promoção de um ideal de saúde, bem-estar e felicidade, muitas vezes alheio ao próprio sujeito. O sujeito é ejetado do discurso sobre seu próprio sofrimento; perde-se o traço particular, a marca do seu estilo.

Oferecer a prioridade lógica ao sofrimento, e não ao saber científico, é uma forma de afirmar que a experiência subjetiva não está fundada apenas nas propriedades biológicas, mas também nas marcas sociossimbólicas e na trajetória existencial singular. Dessa forma, somos desafiados a considerar a maneira singular de interrogar o sofrimento psíquico e a forma com que o sujeito particular inventa modos de se relacionar com o mundo.

Vemos em Canguilhem e em Fulford o compromisso ético de resistir à normalização, pois indicam que a prática terapêutica não visa à fidelidade a um tipo específico ou a normas individuais prévias, mas a aquisição de uma nova norma individual. Entendem que todo vivente humano tem a capacidade de subverter a ordem instituída e criar outra mais saudável para si, seja no âmbito biológico seja no social e, assim, colocam a clínica como dispositivo de resistência às práticas normalizadoras em saúde.

\section{Referências}

Banzato, C.E.M. \& Dantas, C.R. (2012). Lidando com valores no hospital geral. In: N.J. Botega (Org.), Prática psiquiátrica no Hospital Geral (3ª ed., pp. 658-668). Porto Alegre: Artmed. Blackburn, S. (1994). Dicionário Oxford de Filosofia. Rio de Janeiro: Jorge Zahar.

Boorse, C. (1975). On the distinction between disease and illness. Philosophy and Public Affairs, 5, 49-68.

Boorse, C. (1977). Health as a theoretical concept. Philosophy of Science, 44(4), 542-573.

Canguilhem, G. (1995). O normal e o patológico. Rio de Janeiro: Forense Universitária.

De Block, A. \& Adriaens, R.P. (2011). Why philosophers of psyquiatry should care about evolutionary theory. In: R.P. Adriaens \& A. De Block. (Org.), Maladapting minds: philosophy, 
psychiatry, and evolutionary theory (pp. 3-27). New York: Oxford University Press.

Fulford, K.W.M. (1989). Moral theory and medical practice. Cambridge: Cambridge University Press.

Fulford, K.W.M. (1993a). Mental Illness and The Mind-Brain Problem: Delusion, Belief And Searle's Theory of Intentionality. Theoretical Medicine, 14, 181-194.

Fulford, K.W.M. (1993b). Praxis makes perfect: illness as a bridge between biological concepts of disease and social conceptions of health. Theoretical Medicine, 14, 305-320.

Fulford, K.W.M. (2001). What is (mental) disease?: an open letter to Christopher Boorse. Journal of Medical Ethics, 27, 80-85.

Fulford, K.W.M. (2004). Ten Principles of Values-Based Medicine. In J. Radden (Org.), The Philosophy of Psychiatry: A Companion (pp. 205-234). New York: Oxford University Press.

Gayon, J. (1998). The concept of individuality in Canguilhem's philosophy of biology. Journal of the History of Biology, 31, 305-325.

Ghiraldelli Junior, P. (1999). O que há de real e de irreal com o realismo: Searle versus Rorty. Trans/Form/Ação [online], 21-22(1), 119-129.

Giroux, E. (2011). Después de Canguilhem: definir la salud y la enfermedad. Bogota: Universidad El Bosque.

Guerrero, J.D. (2011). Against Naturalist Conceptions of Health: In Defense of Constrained Normativism. (Tese de doutorado em Filosofia). University of Calgary, Calgary.

Klender, K. (2008). Introduction. Why does Psychiatry need Philosophy? In K. Klender, J. Parnas, (Orgs.), Philosophical Issues in Psychiatry. Explanation, Phenomenology and Nosology (pp. 1-16). Baltimore: The Johns Hopkins University Press.

Nordenfelt, L.(2001). Health, Science and Ordinary Language. Amsterdam/New York: Editions Rodopi.

Putnam, H. (1999). O realismo de rosto humano. Lisboa: Instituto Piaget.

Rosenberg, C.E. (2006). Contested Boundaries: psychiatry, disease, and diagnosis. Perspectives in Biology and Medicine, 49(3): 407-424.

Ruse, M. (1997). Defining Disease: the question of sexual orientation. In M.J. Humber \& F.R. Almeder, What is Disease? Totowa: Humana Press Inc.

Searle, J.R. (1995). The construction of social reality. London: Penguin Books.

Woodbridge-Dodd, K. \& Fulford, K.W.M. (2004). Valores de quem? Manual para prática baseada em valores na saúde mental. Warwick: Warwick Medical School.

\section{Resumos}

(The tension between naturalism and normativism in the field of defining diseases)

A trend can be seen in contemporary psychiatry toward reducing the subjective experience of illness to neurophysiological findings. Research by Bill Fulford and Georges Canguilhem goes against this trend. Fulford holds that illness comes before disease, while 


\section{OBSERVANDO A PSIQUIATRIA}

Canguilhem defends the idea that illness precedes disease. They both hold to the inseparability of facts and values. They emphasize the socio-symbolic dimension of subjective experience, thus stressing the importance of clinical approaches for dealing with human suffering.

Key words: Philosophy of medicine, psychiatry, Fulford, Canguilhem

(La tension naturalisme/normativisme dans le domaine de la définition de la maladie)

On observe dans la psychiatrie contemporaine une tendance à réduire l'expérience subjective de la maladie à des découvertes neurophysiologiques. Les travaux de Bill Fulford et de Georges Canguilhem se situent à contrecourant de ce mouvement. Le premier revendique l'antécédence de la illness par rapport à la disease. Canguilhem soutient que le pathos précède le logos et tous deux affirment l'inséparabilité entre les faits et les valeurs. Ils mettent ainsi en avant la dimension socio-symbolique de l'expérience subjective et soulignent la centralité de la clinique pour la réflexion sur la souffrance humaine.

Mots clés: Philosophie de la médecine, psychiatrie, Fulford, Canguilhem

(La tensión naturalismo/normativismo en el campo de la definición de la enfermedad)

Observamos una tendencia de la psiquiatría contemporánea en reducir la experiencia subjetiva de la enfermedad a hallazgos neurofisiológicos. En dirección contraria a ese movimiento están los trabajos de Bill Fulford y Georges Canguilhem. El primero apuesta por la precedencia de la illness sobre la disease; Canguilhem defiende la idea de que el pathos precede al logos y ambos afirman la inseparabilidad entre hechos y valores. Traen, así, el énfasis en la dimensión socio-simbólica de la experiencia subjetiva, marcando la centralidad de la clínica para la investigación del sufrimiento humano.

Palabras claves: Filosofía de la medicina, psiquiatría, Fulford, Canguilhem

(Das Spannungsfeld Naturalismus/Normativismus im Bereich der Definition von Krankheit)

In der heutigen Psychiatrie ist eine Tendenz zu beobachten, die subjektive Erfahrung des Krankseins auf neurophysiologische Entdeckungen zu reduzieren. In entgegengesetzter Richtung entwickeln Bill Fulford und Georges Canguilhem ihre Studien. Fulford setzt auf die Präzedenz der illness vor der disease; Canguilhem vertritt die Auffassung, dass das Pathos dem Logos vorangeht und beide die Untrennbarkeit von Tatsachen und Werten bezeugen. Somit setzen sie den Schwerpunkt auf die sozial-symbolische Dimension der subjektiven Erfahrung und kennzeichnen die Zentralität der Klinik in der Fragestellung zum menschlichen Leiden.

Schlüsselwörter: Philosophie der Medizin, Psychiatrie, Fulford, Canguilhem 
Citação/Citation: Gaudenzi, P. (2014, dezembro). A tensão naturalismo/normativismo no campo da definição da doença. Revista Latinoamericana de Psicopatologia Fundamental, 17(4), 911-924.

Editor do artigo/Editor: Prof. Dr. Claudio E. M. Banzato; Profa. Dra. Rafaela Zorzanelli

Recebido/Received: 12.9.2014/9.12.2014 Aceito/Accepted: 25.10.2014/ 10.25.2014

Copyright: (C) 2009 Associação Universitária de Pesquisa em Psicopatologia Fundamental/ University Association for Research in Fundamental Psychopathology. Este é um artigo de livre acesso, que permite uso irrestrito, distribuição e reprodução em qualquer meio, desde que o autor e a fonte sejam citados / This is an open-access article, which permits unrestricted use, distribution, and reproduction in any medium, provided the original author and source are credited.

Financiamento/Funding: A autora declara não ter sido financiada ou apoiada / The author has no support or funding to report.

Conflito de interesses/Conflict of interest: A autora declara que não há conflito de interesses / The author has no conflict of interest to declare.

\section{Paula Gaudenzi}

Médica; Doutora em Saúde Coletiva; Professora do Instituto Federal do Rio de Janeiro IFRJ (Rio de Janeiro, RJ, Br).

Av. Praia do Flamengo, 378/101

22210-065 Rio de Janeiro, RJ, Br

e-mail: paula.gaudenzi@gmail.com 